\title{
Improved Functionalization of Multiwalled Carbon Nanotubes in Ultra-Low Acid Volume: Effect of Solid/Liquid Interface
}

\author{
Vinícius G. Castro, ${ }^{a, b}$ Ingrid B. Costa, ${ }^{a}$ Felipe S. Medeiros,,${ }^{a, b}$ Éder J. Siqueira, ${ }^{a}$ \\ Alexander H. Kasama, ${ }^{c}$ Kátia C. S. Figueiredo, ${ }^{a, b}$ Rodrigo L. Lavall ${ }^{a}$ and \\ Glaura G. Silva ${ }^{\circledR *, a}$ \\ ${ }^{a}$ Departamento de Química, Instituto de Ciências Exatas, \\ Universidade Federal de Minas Gerais, 31270-901 Belo Horizonte-MG, Brazil \\ ${ }^{b}$ Departamento de Engenharia Química, Escola de Engenharia, \\ Universidade Federal de Minas Gerais, 31270-901 Belo Horizonte-MG, Brazil \\ ${ }^{c}$ Centro de Pesquisas Leopoldo Américo Miguez de Mello (CENPES), Petrobras, \\ 21941-598 Rio de Janeiro-RJ, Brazil
}

\begin{abstract}
A comprehensive study of a less harmful functionalization of multiwalled carbon nanotubes (MWCNT) was performed. The influence of ultrasonic cavitation and solid/liquid interface was indirectly evaluated by statistical characterization of MWCNT aspect ratio and degree of functionalization. MWCNT aspect ratio was determined through hundreds of electron microscopy images. Degree of functionalization of MWCNT was characterized by elemental analysis and thermogravimetry. Distribution of different oxygen-containing functional groups was assessed by potentiometric titration. MWCNT aqueous dispersions were evaluated by electrophoretic mobility and dynamic light scattering. An acid volume over $90 \%$ lower than usually reported in literature guaranteed MWCNTs with significantly larger aspect ratio, higher proportion of carboxylic groups and a more negative zeta potential in aqueous suspension. These results can be related to a more localized ultrasonic cavitation in MWCNTs/acid interface for reduced liquid volumes. This distinguished influence of ultrasonic cavitation may be a valuable contribution to more eco-friendly processes with nanomaterials.
\end{abstract}

Keywords: carbon nanotubes, ultrasonic cavitation, solid/liquid interface, functionalization

\section{Introduction}

Carbon nanotubes (CNTs) are widely studied in numerous applications due to their high aspect ratio combined with excellent mechanical, thermal and electrical properties. ${ }^{1-3}$ However, the numerous van der Waals interactions between CNTs cause a strong agglomeration tendency and consequent formation of micrometric agglomerates. ${ }^{3,4}$ Thus, dispersion in a variety of solvents and polymeric matrices is a critical challenge to ensure that the use of CNTs actually occurs in nanometric scale with effective transfer of their properties to the products of interest.

Chemical modifications of CNT surface comprise the main strategy to enhance their interfacial interaction with other materials. ${ }^{4-6}$ Acid treatments are the most commonly

*e-mail: glaura.goulart@gmail.com, glaura@qui.ufmg.br used method for insertion of covalently bonded functional groups, ${ }^{7-9}$ among other less usual approaches involving ozone ${ }^{10-12}$ oxygen plasma ${ }^{12-14}$ and ultraviolet radiation. ${ }^{15}$ Acid treatments generally use nitric $\left(\mathrm{HNO}_{3}\right)$ and sulfuric $\left(\mathrm{H}_{2} \mathrm{SO}_{4}\right)$ acids in ultrasound, ${ }^{16-24}$ reflux $^{8,9,21-23,25-29}$ or ultrasound followed by reflux, ${ }^{22,30}$ besides the less common use of microwave. ${ }^{31-33}$

A more efficient functionalization via ultrasound in comparison to reflux was observed in some studies. ${ }^{17,21,22}$ The use of ultrasound is related to the phenomenon of acoustic or ultrasonic cavitation, which occurs by the interaction of microbubbles with the generated acoustic field. This cavitation involves mechanisms of stable and inertial types..$^{21,34-37}$ Stable cavitation is based on bubble oscillation, without necessarily promoting their collapse. ${ }^{35-37}$ Inertial cavitation is related to the increase of microbubbles and their subsequent implosion, which 
generates localized zones of high energy dissipation, with heating that may reach thousands of Celsius degrees, high pressure and strong shearing. ${ }^{35-37}$ This phenomenon occurs in milliseconds, with high heating and cooling rates. ${ }^{35}$ Acoustic cavitation promotes reactions in liquids and a more efficient contact between different materials, which motivates its application on functionalization and dispersion of CNTs. ${ }^{21,37}$ Nevertheless, there is a lack of studies about the ultrasonic cavitation mechanism in the functionalization of CNTs.

Acid treatments involve mainly the formation of carboxylic groups, hydroxyls, phenols, lactones, acid anhydrides and quinones. ${ }^{8,21,32,38,39}$ Carboxylic groups are particularly aimed for the introduction of further chemical functions, as amides or esters. ${ }^{5-7,16,30,40}$ Acid treatments also induce the reduction of CNTs length, ${ }^{10,19,20}$ which is prejudicial for applications dependent on higher aspect ratios, for instance the manufacturing of polymeric composites. ${ }^{20,41,42}$ Therefore, functionalization involves a trade-off between two cardinal characteristics of CNTs: structural quality, related with its aspect ratio, and dispersion, dependent on its chemical surface.

CNT fragments are also formed as by-products of the functionalization. These fragments are comprised of polycyclic aromatic structures with oxygenated functions and remain adsorbed to CNTs. ${ }^{26,32,43}$ These materials are commonly reported as debris, ${ }^{9,29-33}$ fulvic acids ${ }^{27,28}$ or carboxylated carbonaceous fragments. ${ }^{29,38,39,43-45}$ The oxygenated functions are not exclusively consisted of carboxylic groups, debris represent a general terminology and fulvic acids refer to substances present in soils. Therefore, the denomination of oxygenated carbonaceous fragments (OCFs) will be used in this work. OCFs alter the CNTs surface characteristics, ${ }^{30,33}$ which may significantly influence the behavior of these nanomaterials, such as their dispersion ${ }^{9,28}$ and electrochemical properties. ${ }^{43}$ Since the interactions between OCFs and multiwalled CNTs (MWCNTs) are relatively weak if compared to the functional groups covalently linked to MWCNT walls, the desorption of OCFs is occasionally performed by a base wash. ${ }^{27,30,32}$ However, depending on the desired application, this step is not mandatory, especially due to the contribution of OCFs for better dispersion and stability of MWCNTs in polar solvents. ${ }^{28}$

An optimized relation between the insertion of oxygenated groups and the preservation of CNT structural quality is one of the main requirements for improved functionalization methods. ${ }^{31} \mathrm{~A}$ more efficient acid consumption is also of great importance for less waste generation and more eco-friendly processes. The most commonly reported range of CNTs per acid volume is
1-10 mg mL ${ }^{-1}, 17,18,20,21,29,30$ which represents the need of hundreds of liters of acid for functionalizing $1 \mathrm{~kg}$ of MWCNTs. Besides that, the ultrasound power declines with increasing distance from the source, which is more pronounced in large equipment and generates difficulties for homogenous processing. ${ }^{46}$ Therefore, smaller processing volumes in ultrasound are also important for scaling up without compromising the functionalization results.

The goal of the present work was to establish a better understanding of solid/liquid interface role in ultrasonicassisted functionalization of MWCNTs, with the aim of enabling an improved MWCNT chemical modification and less waste generation. A study of temperature, time and a critical reduction in acid volume was performed in the presence of ultrasonic cavitation. The functionalized MWCNTs were characterized by elemental analysis (EA), thermogravimetry (TGA), potentiometric titration, scanning electron microscopy (SEM), transmission electron microscopy (TEM), electrophoretic mobility and dynamic light scattering (DLS). These work findings may be applied towards MWCNTs with enhanced structural preservation, as well as more efficient and environmentally friendly processes.

\section{Experimental}

\section{Materials}

MWCNTs were provided by CTNano, Belo Horizonte, Brazil, with average diameter of $19 \mathrm{~nm}$, length distribution up to $30 \mu \mathrm{m}$, average length of $6 \mu \mathrm{m}$ by arithmetic mean and $12 \mu \mathrm{m}$ by weighted mean. Sulfuric and nitric acids were acquired from Synth, São Paulo, Brazil.

\section{Functionalization of MWCNTs}

The functionalization of MWCNTs was conducted with a solution of $\mathrm{H}_{2} \mathrm{SO}_{4} / \mathrm{HNO}_{3}$ (3:1 in volume) inserted in a volumetric flask, which was immersed in an ultrasonic bath (frequency of $37 \mathrm{kHz}$ ). All treatments were performed with $3 \mathrm{~g}$ of MWCNTs and mechanical stirring of $430 \mathrm{rpm}$ to homogenize the system. Temperature and reaction time were initially varied from $50{ }^{\circ} \mathrm{C}$ and $90 \mathrm{~min}$ to $70{ }^{\circ} \mathrm{C}$ and $20 \mathrm{~min}$, using a fixed acid volume of $176 \mathrm{~mL}$, based on previous experiments. ${ }^{24}$ The reduction of acid volume was subsequently varied from 176 to 88,44 and $20 \mathrm{~mL}$, using fixed conditions of $70{ }^{\circ} \mathrm{C}$ and $20 \mathrm{~min}$. Washing and separation of functionalized MWCNTs were conducted via filtration and continuous addition of deionized water in a sintered glass funnel with pore diameter of 10-16 $\mu \mathrm{m}$ until $\mathrm{pH}$ 7. Drying was performed in an oven at $100^{\circ} \mathrm{C}$ for $8 \mathrm{~h}$. 


\section{Extraction of OFCs}

Fractions of each functionalized MWCNT sample were submitted to $1 \mathrm{~h}$ of reflux in $\mathrm{NaOH} 1.0 \mathrm{~mol} \mathrm{~L}^{-1}$ solution for extraction of OCFs, washed with deionized water until $\mathrm{pH} 7$ and filtered in polytetrafluoroethylene (PTFE) membrane with pore diameter of $0.45 \mu \mathrm{m}$. The retained MWCNTs were refluxed in $1.0 \mathrm{~mol} \mathrm{~L}^{-1} \mathrm{HCl}$ solution for reprotonation of their acid sites, washed with deionized water and filtered in PTFE membrane. The MWCNTs were then dried in an oven at $100{ }^{\circ} \mathrm{C}$ for $4 \mathrm{~h}$.

\section{Sample characterization}

EA and TGA measurements were performed before and after OCF removal. EA was conducted on Thermo Flash 2000 Analyser and the results correspond to the average of 3 measurements for each sample. TGA analysis was performed in duplicate on Q5000 (TA Instruments), with heating rate of $5{ }^{\circ} \mathrm{C} \mathrm{min} \mathrm{m}^{-1}$, from 30 to $900{ }^{\circ} \mathrm{C}$, in synthetic air atmosphere and flow rate of $25 \mathrm{~mL} \mathrm{~min}^{-1}$.

Potentiometric titration was based on the methodology of Kim et al ${ }^{47}$ for the evaluation of the surface composition of MWCNTs after OCF removal and the results correspond to the average of three measurements for each sample. The MWCNTs were stirred for $30 \mathrm{~min}$ in ultrasonic bath with $0.01 \mathrm{~mol} \mathrm{~L}^{-1}$ solutions of different bases $\left(\mathrm{NaOH}, \mathrm{Na}_{2} \mathrm{CO}_{3}\right.$, $\mathrm{NaHCO}_{3}$ ), in sealed bottles with $0.1 \mathrm{~mol} \mathrm{~L}^{-1} \mathrm{NaCl}$, followed by centrifugation for $20 \mathrm{~min}$ at $4000 \mathrm{rpm}$. Supernatant aliquots of $5 \mathrm{~mL}$ were titrated by $0.01 \mathrm{~mol} \mathrm{~L}^{-1} \mathrm{HCl}$ solution in $0.1 \mathrm{~mol} \mathrm{~L}^{-1} \mathrm{NaCl}$, with $\mathrm{pH}$ monitoring by a Hanna $\mathrm{pH} 20$ instrument.

SEM images for the MWCNTs were carried out on a cannon emission field effect Quanta 200-FEG/FEI and TEM images on a FEI TECNAI G2 microscope, before OCF removal. The preparation of the samples for both microscopy techniques was conducted by sonication of MWCNTs in isopropyl alcohol and posterior deposition onto 200 mesh carbon/copper grids. Determination of length distribution was assessed by ImageJ software. ${ }^{48}$ Approximately 170 MWCNTs were measured by SEM images and 140 MWCNTs by TEM images for each sample in order to obtain statistical results with high significance, totalizing the measurement of more than two thousand MWCNTs by electronic microscopy.

Zeta $(\zeta)$ potential values of aqueous suspensions of MWCNTs in concentration of $0.01 \%\left(\mathrm{~m} \mathrm{v}^{-1}\right)$, before and after OCF removal, were obtained by electrophoretic mobility technique. Hydrodynamic diameter (HD) values were determined by measurements of DLS in a $173^{\circ}$ angle for the same suspensions. The samples were prepared by sonication of the MWCNTs in deionized water for $30 \mathrm{~min}$. Aliquots from the supernatants of the dispersions after $72 \mathrm{~h}$ at rest were analyzed on Zetasizer Nanoseries (Malvern Instruments). The results from both techniques represent the arithmetic mean of approximately 60 measurements carried out for each sample at $25^{\circ} \mathrm{C}$.

\section{Results and Discussion}

Acid volume and temperature effects on degree of functionalization

Table 1 presents the results of degree of functionalization (mass \%) obtained from EA and TGA analysis. The degree of total functionalization (related both to covalent groups and OCFs) and the degree of covalent functionalization (only related to groups covalently linked to MWCNTs) were determined considering samples before and after OCF removal, respectively. The degree of functionalization is composed mainly by oxygen, besides a small amount of hydrogen, nitrogen and sulfur content according do EA. The percentage of each element is shown in Table S1 (Supplementary Information (SI) section).

The degrees of functionalization (total and covalent) were not altered with the reduction of treatment time from 90 to 20 min along with the raise in temperature from 50 to $70{ }^{\circ} \mathrm{C}$. Therefore, a moderate temperature increase to $70{ }^{\circ} \mathrm{C}$ (value lower than those over $100{ }^{\circ} \mathrm{C}$ used in treatments based on reflux) allowed an expressive functionalization in just 20 min of reaction via ultrasound, which represents a reduction of almost $80 \%$ in time spent on the process.

The degree of functionalization was also maintained, according to experimental deviations, after variation of acid from 176 until $44 \mathrm{~mL}$. The use of $20 \mathrm{~mL}$ did not promote the functionalization to the same extent, which can be related to an insufficient contact between the MWCNTs and acid medium in this condition. Functionalization of $3 \mathrm{~g}$ of MWCNTs with $44 \mathrm{~mL}$ of acid is equivalent to a proportion of approximately $70 \mathrm{mg} \mathrm{mL}^{-1}$. This represents a reduction over $90 \%$ on the consumption of acids in comparison to the average value of $5 \mathrm{mg} \mathrm{mL}^{-1}$ reported by the literature. ${ }^{17,18,20,21,29,30}$ Cho et al. ${ }^{9}$ evaluated a ratio up to $50 \mathrm{mg} \mathrm{mL}^{-1}$ in acid treatment of MWCNTs via reflux, however, the degree of functionalization was maintained only with ratios up to $12.5 \mathrm{mg} \mathrm{mL}^{-1}$. The improved methodology proposed herein promoted a critical reduction in acid volume without altering the extent of functionalization.

The percentage of covalent functionalization was calculated from the ratio of the values obtained for samples before and after OCF removal. These results 
Table 1. Degrees of functionalization based on EA and TGA analysis of acid treated MWCNTs

\begin{tabular}{|c|c|c|c|c|}
\hline Treatment & Type of analysis & $\begin{array}{c}\text { Degree of total } \\
\text { functionalization / mass } \%\end{array}$ & $\begin{array}{c}\text { Degree of covalent } \\
\text { functionalization / mass } \%\end{array}$ & $\begin{array}{c}\text { Ratio of covalent } \\
\text { functionalization } / \%\end{array}$ \\
\hline \multirow[t]{2}{*}{$50^{\circ} \mathrm{C}, 90 \mathrm{~min}, 176 \mathrm{~mL}$} & EA & $7.5 \pm 0.1$ & $4.7 \pm 0.0$ & $63 \pm 1$ \\
\hline & TGA & $7.8 \pm 0.1$ & $4.5 \pm 0.3$ & $58 \pm 4$ \\
\hline \multirow[t]{2}{*}{$70{ }^{\circ} \mathrm{C}, 20 \mathrm{~min}, 176 \mathrm{~mL}$} & EA & $7.5 \pm 0.2$ & $4.8 \pm 0.0$ & $64 \pm 2$ \\
\hline & TGA & $7.6 \pm 0.8$ & $5.1 \pm 0.1$ & $67 \pm 7$ \\
\hline \multirow[t]{2}{*}{$70{ }^{\circ} \mathrm{C}, 20 \mathrm{~min}, 88 \mathrm{~mL}$} & EA & $7.3 \pm 0.3$ & $4.6 \pm 0.1$ & $63 \pm 3$ \\
\hline & TGA & $7.6 \pm 0.0$ & $4.4 \pm 0.6$ & $58 \pm 8$ \\
\hline \multirow[t]{2}{*}{$70{ }^{\circ} \mathrm{C}, 20 \mathrm{~min}, 44 \mathrm{~mL}$} & EA & $7.9 \pm 0.8$ & $4.5 \pm 0.1$ & $57 \pm 6$ \\
\hline & TGA & $7.7 \pm 0.3$ & $5.0 \pm 0.3$ & $65 \pm 6$ \\
\hline \multirow[t]{2}{*}{$70{ }^{\circ} \mathrm{C}, 20 \mathrm{~min}, 20 \mathrm{~mL}$} & EA & $5.6 \pm 0.3$ & $4.0 \pm 0.3$ & $71 \pm 6$ \\
\hline & TGA & $5.9 \pm 0.1$ & $3.5 \pm 0.4$ & $59 \pm 7$ \\
\hline
\end{tabular}

EA: elemental analysis; TGA: termogravimetry analysis.

are also presented on Table 1 . The average covalent ratio observed for the functionalized MWCNTs was $64 \%$ and no variation trend was observed within the experiment deviations. Wu and Mitra $^{32}$ reported percentages from 47 to $67 \%$ depending on the type of MWCNTs used for functionalization via microwave. The value of $47 \%$ was reported for functionalization of MWCNTs with length of 10-30 $\mu \mathrm{m}$ and diameter of $10-20 \mathrm{~nm}$, the most similar characteristics to the MWCNTs employed in this study. The high proportion of covalent functionalization achieved in the present work is a desirable factor for further chemical modifications of MWCNTs and covalent linkage with polymers.

Figure 1 shows the TGA curves for MWCNTs functionalized at $70{ }^{\circ} \mathrm{C}, 20 \mathrm{~min}$ and intermediate acid volume condition $(88 \mathrm{~mL}$ ), besides the TGA curves for pristine MWCNTs. All other samples present a similar thermogravimetric behavior, with variations only in the extent of mass loss values.

Studies of thermal degradation of functionalized MWCNTs by TGA in synthetic air atmosphere usually report three main events. The first one is related to the removal of adsorbed water, the second one to the decomposition of present functional groups and the third one to the MWCNT degradation. Mazov et al. ${ }^{25}$ for example, reported events of mass losses in temperatures ranging from 30 to $130{ }^{\circ} \mathrm{C}, 130-450{ }^{\circ} \mathrm{C}$ and above $450{ }^{\circ} \mathrm{C}$. Although the second event is commonly used for calculating the degree of covalent functionalization, ${ }^{25,29,49,50}$ its interpretation is more complex due to the decomposition of functional groups from both MWCNTs and OCFs.

A displacement on the derivative of mass loss after $350{ }^{\circ} \mathrm{C}$ is noticed for the sample in Figure 1, which suggests a preferential decomposition of functional groups

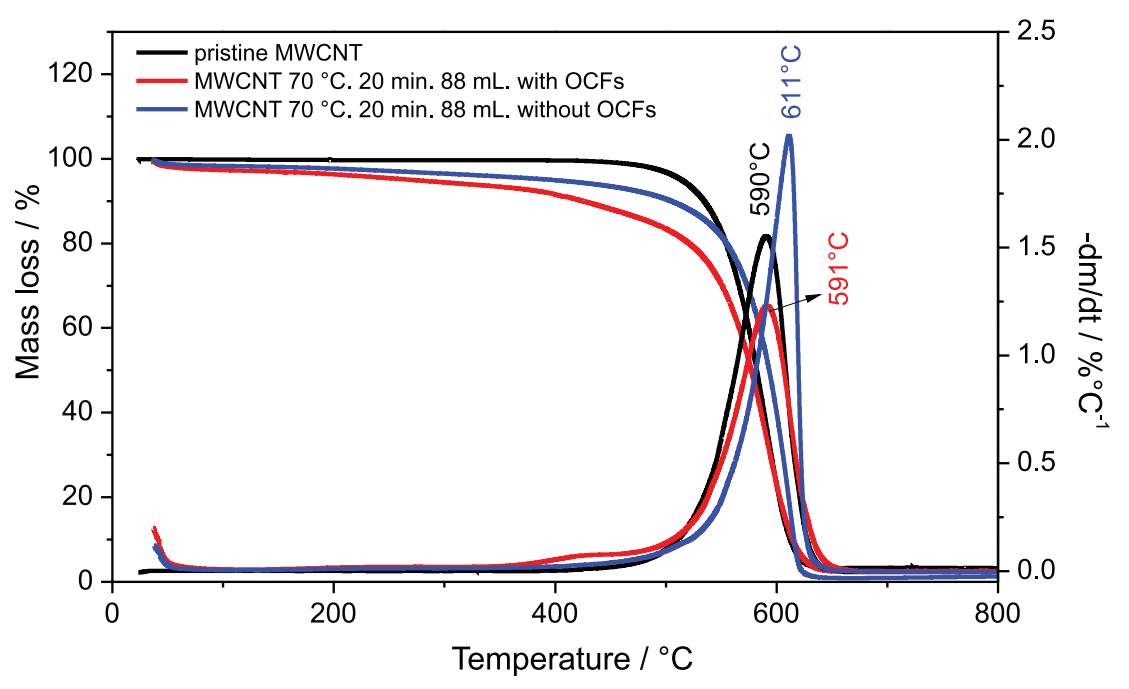

Figure 1. TGA analysis of MWCNTs treated at $70{ }^{\circ} \mathrm{C}, 20 \mathrm{~min}$ and $88 \mathrm{~mL}$ of acid, before and after OCF extraction. 
linked to OCFs starting on this temperature. Nevertheless, a significant mass loss relative to the decomposition of covalent groups after $350{ }^{\circ} \mathrm{C}$ is still present for this sample after the OCF extraction. Therefore, these events cannot be precisely separated by a specific temperature, which requires a characterization before and after OCF extraction. It is also worth noticing that the temperature of maximum degradation increases in $20{ }^{\circ} \mathrm{C}$ after OCF removal. This is an average variation observed for all samples, indicating that the removal of these fragments promotes a better thermal stability to the MWCNTs. In this work, the degree of total functionalization (samples with OCFs) was determined by mass loss between 120 and $430{ }^{\circ} \mathrm{C}$, temperature in which the mass variation rate increases due to OCF desorption/decomposition and subsequent beginning of MWCNT degradation. The degree of covalent functionalization (samples without OCFs) was determined by mass loss of MWCNTs between 120 and $450{ }^{\circ} \mathrm{C}$ due to its higher thermal stability and the absence of any interference of OCF degradation. The obtained values are very similar to the results of EA, which indicate that the designed methodology adequately represents the characteristics of functionalized MWCNTs.

Mass losses of only 0.3 and $0.6 \%$ were noticed by TGA (Figure 1) for pristine MWCNTs in temperatures ranging from 120 to $430{ }^{\circ} \mathrm{C}$ and $120-450{ }^{\circ} \mathrm{C}$, respectively. These data can be associated with minor presence of OCFs, amorphous carbon or MWCNTs with early thermal oxidation in raw material..$^{51}$ Therefore, the majority of oxygenated groups for acid treated samples can be related with the functionalization process.

A residue of 2 mass \% in average was observed for all samples and can be associated with metal catalyst impurities. Acid treatments are known for being able to remove remaining metal catalysts, which can be easily eliminated when are located along the surface of outermost MWCNT walls. ${ }^{52}$ However, the removal of internally confined metal impurities has proved to be a great challenge, as Suryanto et al. ${ }^{52}$ pointed out. Therefore, it is common to consider a range of 0.5-2.0 mass\% of residue as high quality MWCNTs..$^{52}$ The production of MWCNTs with trace amounts of metallic impurities generally requires a previous step of annealing. ${ }^{52,53}$ Alternatively, very harsh oxidative conditions can expose the internal metallic impurities through greater damage of MWCNTs. ${ }^{52,53}$ Andrade et al. ${ }^{29}$ reported a metallic residue around 2.5 mass $\%$ after acid treatment at $75{ }^{\circ} \mathrm{C}$, a similar value to results obtained in this work. The same authors reported a metallic residue less than 0.5 mass $\%$ after a treatment at $175{ }^{\circ} \mathrm{C} .{ }^{29}$ Therefore, depending on desired applications, a previous step of annealing is indicated to remove encapsulated metal catalysts without additional damage to MWCNTs.

Morphological study and correlation with ultrasonic cavitation

MWCNT length distribution was determined by measuring approximately 170 nanotubes for each sample (in SEM images, such as that presented in Figure 2b). Even though the discussion about length in some papers are conducted by images with the presence of many MWCNTs (as Figure 2a), a correct statistical evaluation of their values demands observation from the beginning up to the end of isolated tubes (Figure 2b). Just by way of illustration, extra SEM micrographs are presented on Figure S1 (SI section), which exemplifies the images used in this work to obtain statistical results with the high significance described herein.
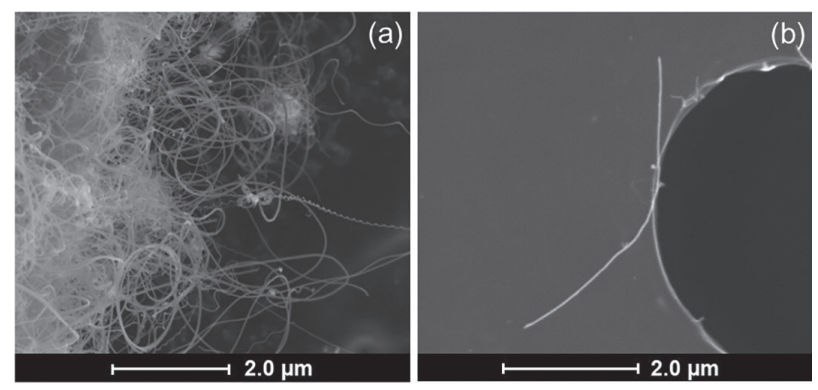

Figure 2. SEM images of (a) agglomerated MWCNTs and (b) isolated MWCNTs after functionalization at $70{ }^{\circ} \mathrm{C}, 20 \mathrm{~min}$ and $44 \mathrm{~mL}$ of acid volume.

The length distribution for pristine and functionalized MWCNTs (with OCFs) can be seen in Figure 3. Pristine MWCNTs exhibit length up to $30 \mu \mathrm{m}$, but graphics were plotted with values up to $10 \mu \mathrm{m}$ for better comparison with functionalized samples. A more significant variation of length distribution was observed for conditions with higher acid volume and lower temperature combined with longer time, especially due to a lower presence of MWCNTs with length greater than $4 \mu \mathrm{m}$ and a higher presence of MWCNTs with length less than $1 \mu \mathrm{m}$. The length distribution profile is very similar for the MWCNTs obtained by acid treatments conducted under the same conditions, as can be observed in Figure S2 (SI section).

Table 2 shows arithmetic and weighted means calculated for MWCNTs submitted to the different acid treatments. The equations used for determination of both means are presented in Supplementary Information.

The accuracy of this characterization method was assessed for MWCNTs functionalized with acid volume of $44 \mathrm{~mL}$ through the execution of three other treatments 

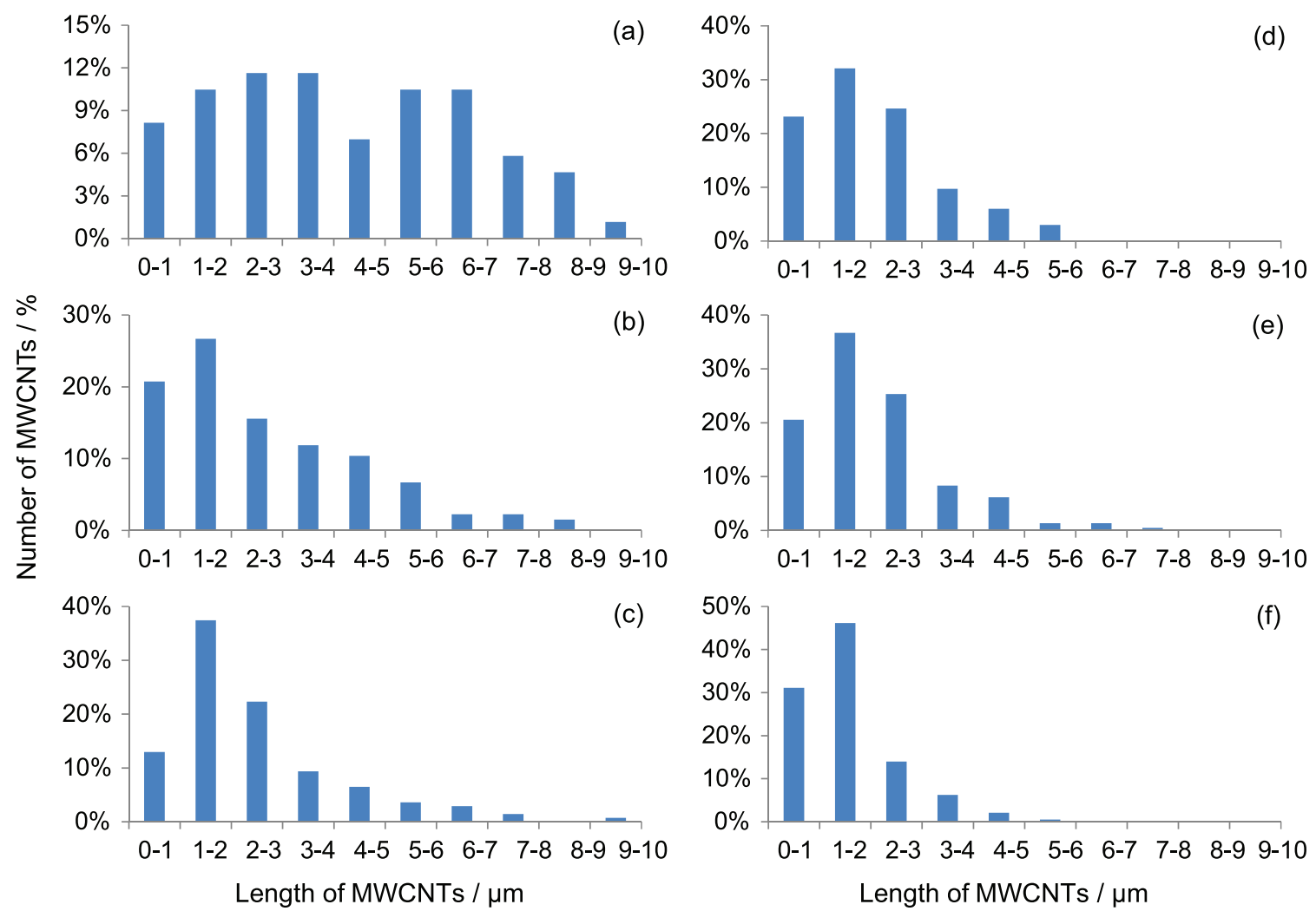

Figure 3. Length distribution of (a) pristine MWCNTs and modified MWCNTs after treatment at (b) $70{ }^{\circ} \mathrm{C}, 20 \mathrm{~min}$ and $20 \mathrm{~mL}$; (c) $70{ }^{\circ} \mathrm{C}, 20 \mathrm{~min}$ and $44 \mathrm{~mL}$; (d) $70{ }^{\circ} \mathrm{C}, 20 \mathrm{~min}$ and $88 \mathrm{~mL}$; (e) $70{ }^{\circ} \mathrm{C}, 20 \mathrm{~min}$ and $176 \mathrm{~mL}$; (f) $50{ }^{\circ} \mathrm{C}, 90 \mathrm{~min}$ and $176 \mathrm{~mL}$.

Table 2. Average length of functionalized MWCNTs

\begin{tabular}{lccc}
\hline Treatment & Arithmetic mean of length / $\mu \mathrm{m}$ & Weighted mean of length / $\mu \mathrm{m}$ & Number of measured MWCNTs \\
\hline- & 6.4 & 11.5 & 172 \\
$50{ }^{\circ} \mathrm{C}, 90 \mathrm{~min}, 176 \mathrm{~mL}$ & 1.5 & 2.2 & 194 \\
$70{ }^{\circ} \mathrm{C}, 20 \mathrm{~min}, 176 \mathrm{~mL}$ & 2.0 & 2.9 & 230 \\
$70{ }^{\circ} \mathrm{C}, 20 \mathrm{~min}, 88 \mathrm{~mL}$ & 2.3 & 3.6 & 136 \\
$70^{\circ} \mathrm{C}, 20 \mathrm{~min}, 44 \mathrm{~mL}$ & 2.8 & 4.5 & 568 \\
$70{ }^{\circ} \mathrm{C}, 20 \mathrm{~min}, 20 \mathrm{~mL}$ & 2.8 & 4.6 & 137 \\
\hline
\end{tabular}

MWCNTs: multiwalled carbon nanotubes.

on the same conditions, with subsequent analysis by SEM and measurement of approximately 140 MWCNTs for each sample (Figure S2, SI section). The standard deviation for both arithmetical and weighted means of MWCNT lengths was $\pm 0.2 \mu \mathrm{m}$, which highlights the difference of length means for treatments at conditions of higher acid volume or temperature.

It is possible to observe that time reduction to $20 \mathrm{~min}$ combined with temperature increase to $70{ }^{\circ} \mathrm{C}$ ensured better preservation of MWCNT length. The raise of temperature is a known strategy to increase functionalization of MWCNTs, ${ }^{8,29}$ but it can compromise their aspect ratio and cause yield product decrease. ${ }^{8}$ These results indicate that acid treatment with moderate temperature and reduced time of exposure to ultrasonic cavitation time is an effective strategy for obtaining expressive functionalization of MWCNTs with better preservation of its length.

Functionalized MWCNTs with higher length were also achieved by decrease in the acid volume. Cho et al..$^{9}$ obtained functionalized MWCNTs with better length preservation by reducing the amount of acid in treatments via reflux, but the degree of functionalization was also reduced according to TGA results. The improved methodology proposed herein promotes a better preservation of MWCNTs without compromising their degree of functionalization, which represents a relevant novelty for the scientific literature. The 


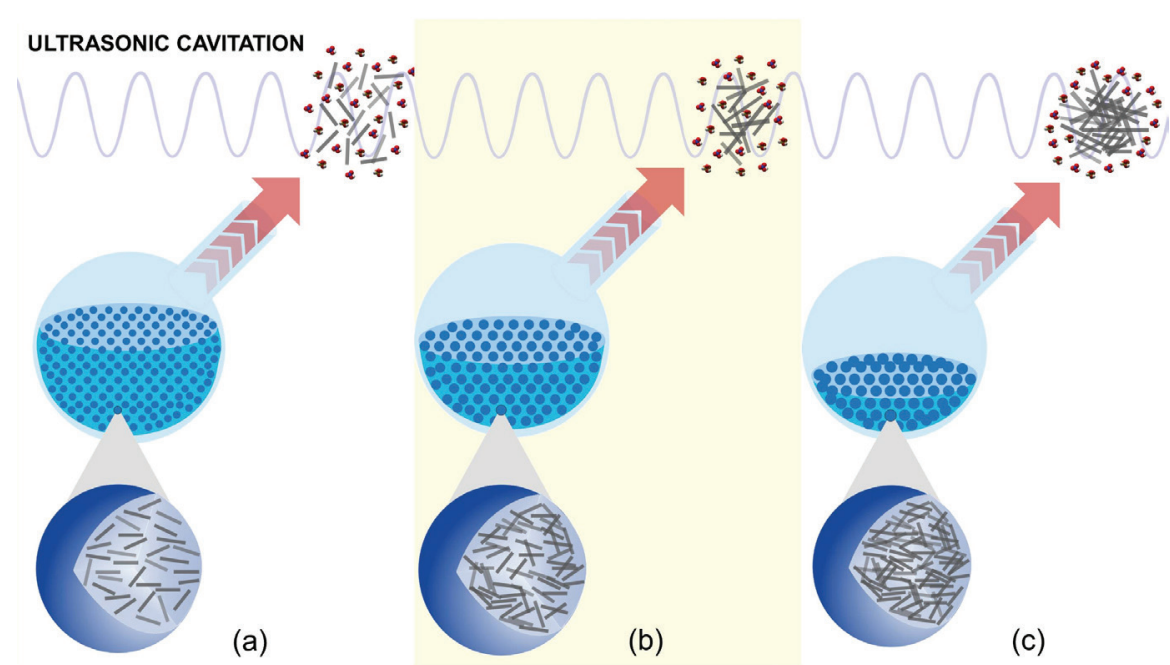

Figure 4. Illustrative scheme of ultrasonic cavitation influence on the functionalization of $3 \mathrm{~g}$ of MWCNTs with different acid volumes: (a) $176 \mathrm{~mL}$, acid in excess; (b) $44 \mathrm{~mL}$, optimal condition; (c) $20 \mathrm{~mL}$, insufficient acid.

optimal conditions of $70{ }^{\circ} \mathrm{C}, 20 \mathrm{~min}$ and $44 \mathrm{~mL}$ resulted in a length $40 \%$ greater by arithmetic mean and $55 \%$ greater by weighted mean in comparison to the treatment at $70^{\circ} \mathrm{C}$, $20 \mathrm{~min}$ and $176 \mathrm{~mL}$. These conditions represent a length around $100 \%$ greater if compared to the treatment at $50{ }^{\circ} \mathrm{C}$, $20 \mathrm{~min}$ and $176 \mathrm{~mL}$.

These outcomes can be related to the phenomenon of ultrasonic cavitation, which promotes MWCNT degradation, especially by the mechanism of inertial cavitation through microbubbles present in the liquid. ${ }^{37}$ Figure 4 illustrates the influence of cavitation in the MWCNTs-acid interface for different acid volumes. MWCNTs are completely surrounded by liquid in larger acid volumes and exposed to ultrasonic cavitation in a greater extension, which may generate a more severe treatment and enhanced rupture of nanotubes (Figure 4a). With reduction of acid volume, solid/ liquid contact is restrained in the face of an increased solid/ solid contact, and cavitation can occur in a more localized way, with better preservation of MWCNT length (Figure 4b). When the acid volume is reduced beyond a minimum amount necessary to the solid/liquid contact, there is no interaction of some MWCNTs with the acid, thus the functionalization is only partial (Figure 4c).

Figure 5 shows representative TEM images of pristine MWCNTs (Figure 5a) and modified MWCNTs (with OCFs) after treatment at $70{ }^{\circ} \mathrm{C}, 20 \mathrm{~min}$ and $44 \mathrm{~mL}$ of acid (Figure $5 \mathrm{~b}$ ). The diameter was determined by measuring around 170 pristine MWCNTs and 130 modified MWCNTs after each functionalization condition. The pristine MWCNTs exhibit an average diameter of $19 \mu \mathrm{m}$, which was not altered and remained at $19 \pm 1 \mu \mathrm{m}$ after all the performed acid treatments. The literature ${ }^{9}$ reports that the oxidation in liquid phase preferentially occurs on the existing defects of MWCNTs and on their edges, but erosion of their walls is also possible. These results indicate that the functionalization of MWCNTs in acid medium via ultrasound did not promoted erosion of MWCNT walls in a sufficient level for the decrease in the diameter. Nevertheless, it can be noticed an alteration of MWCNT structural quality after acid treatment, besides a major presence of adsorbed particles onto its surface (Figure 5). A change in MWCNT integrity is expected for an expressive functionalization of the material and these results have also been reported in other functionalization studies. ${ }^{8,24}$ The material around MWCNT sidewalls after functionalization are commonly associated with the formation of OCFs. ${ }^{8,24}$ Although small adsorbed particles can be observed over CNT walls in raw sample, it is a minor presence and for few tubes, as noticed in extra TEM micrographs for pristine and functionalized CNTs (Figure S3, SI section). Similar images of raw MWCNT surrounded by some defective material are reported in literature, ${ }^{17,26}$ which is generally associated with amorphous carbon originated from the CNT synthesis.

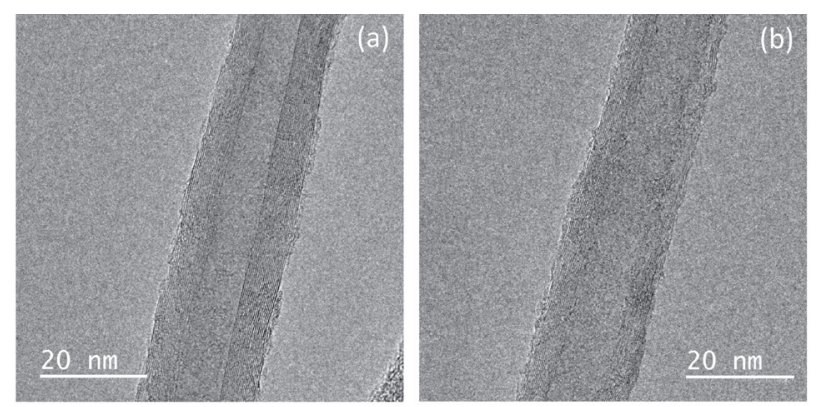

Figure 5. TEM images of (a) pristine MWCNTs and (b) functionalized MWCNTs at $70{ }^{\circ} \mathrm{C}, 20 \mathrm{~min}$ and $44 \mathrm{~mL}$ of acid volume.

The MWCNT aspect ratio can be determined from the diameter and length data. Figure 6 shows the MWCNT aspect ratio (based on weighted mean of length) and degree 


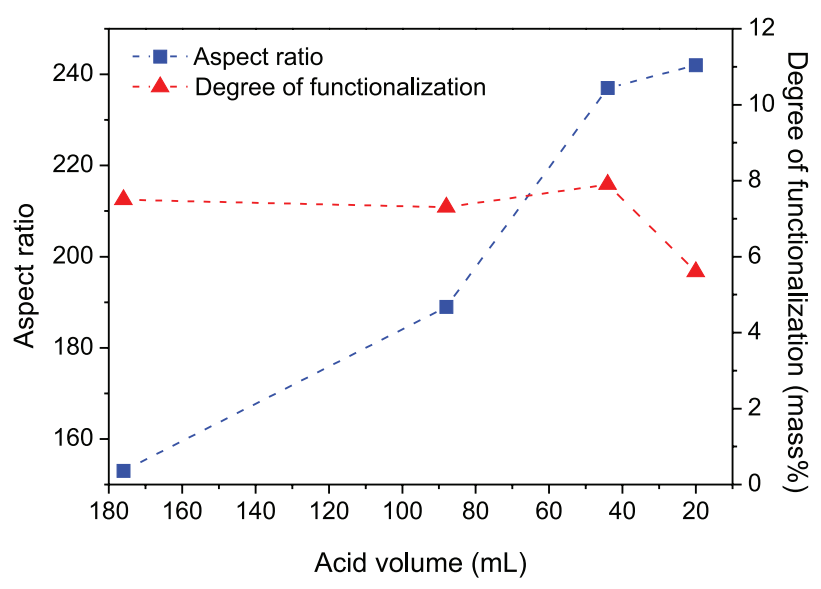

Figure 6. MWCNT aspect ratio and degree of functionalization as a function of acid volume for treatments at $70{ }^{\circ} \mathrm{C}$ and $20 \mathrm{~min}$.

of functionalization (determined EA) versus acid treatment volumes.

From Figure 6, it is noticed that the aspect ratio increases with reduction of volume and reaches a level superior to 200 for the treatment with $44 \mathrm{~mL}$ of acids. Values greater than 200 (for the MWCNT aspect ratio) are desirable to improve the performance of several applications and are particularly relevant for the manufacturing of polymeric composites. ${ }^{9,20,41,43}$ The MWCNTs functionalized with $20 \mathrm{~mL}$ of acid presented a slightly positive oscillation on their aspect ratio in comparison to the condition of $44 \mathrm{~mL}$ (237 to 242), but not exceeding the experimental deviations. Besides that, the treatment with $20 \mathrm{~mL}$ resulted in a decrease of about $25 \%$ in the degree of functionalization compared to the condition of $44 \mathrm{~mL}$ (7.9 to 5.6). Therefore, the treatment at $70{ }^{\circ} \mathrm{C}, 20 \mathrm{~min}$ and $44 \mathrm{~mL}$ presents the best relation of degree of functionalization and aspect ratio for the functionalized MWCNTs, which highlights the optimal solid/liquid interface exposed to ultrasonic cavitation in these conditions.

Impact of MWCNTs/acid interface on functional groups distribution

The results of potentiometric titration for functionalized MWCNTs after OCF extraction can be seen in Figure 7. This analysis was used to characterize carboxylic, lactonic and phenolic covalent groups.

The presence of carboxylic groups is especially relevant to the dispersion of MWCNTs in polar solvents and to allow further chemical reactions. Thus, a high proportion of these functions represents an important factor for MWCNT application. The proportion of carboxyls to the overall functional groups was higher for MWCNTs treated with low acid volume (44 and $20 \mathrm{~mL}$ ). This ratio was $57 \%$ for the MWCNTs functionalized with

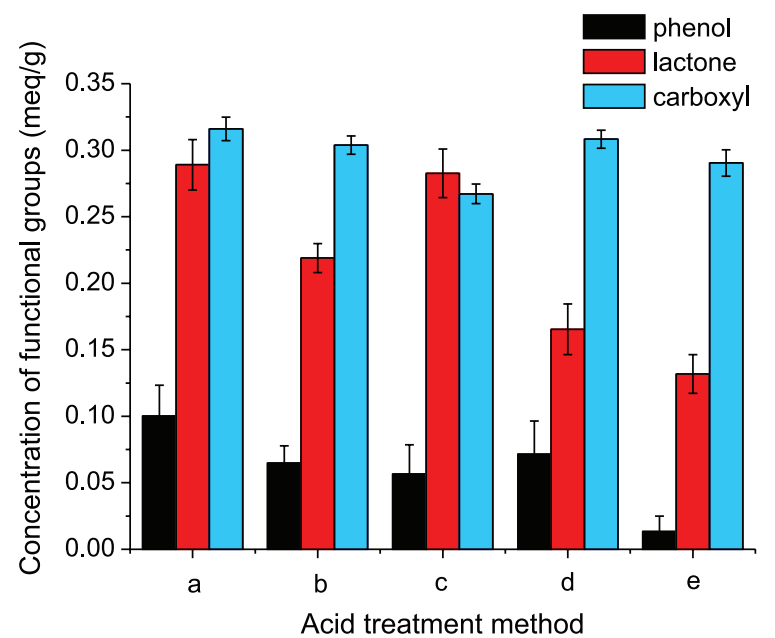

Figure 7. Potentiometric titration results of MWCNTs after OCF extraction for the samples submitted to treatments at: (a) $50{ }^{\circ} \mathrm{C}, 90 \mathrm{~min}$ and $176 \mathrm{~mL}$; (b) $70^{\circ} \mathrm{C}, 20 \mathrm{~min}$ and $176 \mathrm{~mL}$; (c) $70^{\circ} \mathrm{C}, 20 \mathrm{~min}$ and $88 \mathrm{~mL}$; (d) $70{ }^{\circ} \mathrm{C}, 20 \mathrm{~min}$ and $44 \mathrm{~mL}$; (e) $70{ }^{\circ} \mathrm{C}, 20 \mathrm{~min}$ and $20 \mathrm{~mL}$.

acid volume of $44 \mathrm{~mL}$, for instance, which is superior to the range of 44 to $52 \%$ obtained for MWCNTs treated with higher acid amounts. These results are related with a smaller quantity of other functional groups for MWCNTs treated with low acid volume, especially lactones for the condition of $44 \mathrm{~mL}$ and both lactones and phenolic for the condition of $20 \mathrm{~mL}$.

Some papers ${ }^{10,28,30,38}$ report that the insertion of carboxylic groups in MWCNTs occurs after the generation of phenols and lactones, which are formed along the MWCNT surface and in the moment of its rupture. Since higher acid volume results in more aggressive cavitation, a continued generation of phenolic and lactonic groups is possible due to the enhanced rupture of MWCNTs, with no enough time for their conversion to carboxyls. The use of more effective ultrasonic cavitation in lower acid volumes promoted a decreased rupture of MWCNTs, which may prevent a continuous generation of phenolic and lactonic groups, tending toward a higher proportion of carboxylic functions. Furthermore, the treatment at $20 \mathrm{~mL}$ did not result in the same extent of reaction due to the insufficient contact between MWCNTs and acid, which corroborates the optimal condition for the treatment with $44 \mathrm{~mL}$.

\section{Aqueous suspensions evaluation}

Table 3 shows the values of zeta $(\zeta)$ potential and HD for $0.01 \% \mathrm{~m} \mathrm{v}^{-1}$ aqueous suspensions of functionalized MWCNTs.

All suspensions showed $\zeta$ potential values between -38 and $-46 \mathrm{mV}$, which are considerably high in modulus. It has been reported that particles with $\zeta$ higher than $+15 \mathrm{mV}$ or lower than $-15 \mathrm{mV}$ can be stabilized due to electrostatic 
Table 3. Zeta potential and hydrodynamic diameter for $0.01 \% \mathrm{~m} \mathrm{v}^{-1}$ aqueous suspensions of functionalized MWCNTs, before and after OCF extraction

\begin{tabular}{|c|c|c|c|c|}
\hline Treatment & $\begin{array}{l}\zeta \text { potential with } \\
\text { OCFs / mV }\end{array}$ & $\begin{array}{c}\zeta \text { potential without } \\
\text { OCFs / mV }\end{array}$ & HD with OCFs / nm & $\mathrm{HD}$ without OCFs / nm \\
\hline $50^{\circ} \mathrm{C}, 90 \mathrm{~min}, 176 \mathrm{~mL}$ & $-46.0 \pm 0.7$ & $-43.6 \pm 0.8$ & $279.4 \pm 5.1$ & $323.4 \pm 5.6$ \\
\hline $70^{\circ} \mathrm{C}, 20 \mathrm{~min}, 176 \mathrm{~mL}$ & $-46.0 \pm 0.6$ & $-41.0 \pm 0.9$ & $367.8 \pm 46.7$ & $368.8 \pm 46.0$ \\
\hline $70{ }^{\circ} \mathrm{C}, 20 \mathrm{~min}, 88 \mathrm{~mL}$ & $-44.0 \pm 0.7$ & $-38.5 \pm 0.3$ & $302.4 \pm 7.0$ & $322.2 \pm 9.6$ \\
\hline $70^{\circ} \mathrm{C}, 20 \mathrm{~min}, 44 \mathrm{~mL}$ & $-46.6 \pm 0.4$ & $-44.8 \pm 0.5$ & $297.6 \pm 8.4$ & $303.3 \pm 5.2$ \\
\hline $70^{\circ} \mathrm{C}, 20 \mathrm{~min}, 20 \mathrm{~mL}$ & $-43.7 \pm 0.4$ & $-40.1 \pm 0.9$ & $351.0 \pm 29.8$ & $413.1 \pm 26.8$ \\
\hline
\end{tabular}

$\zeta$ potential: zeta potential; OCFs: oxygenated carbonaceous fragments; HD: hydrodynamic diameter.

repulsion interactions..$^{54,55} \mathrm{~A} \zeta$ larger than $30 \mathrm{mV}$ (in modulus) is generally considered to represent sufficient interparticle electrostatic repulsion, ensuring a stable dispersion. ${ }^{18,56}$ The dispersion quality are expected to scale with the zeta potential. ${ }^{57}$ Therefore, the obtained results indicate a good stability of functionalized MWCNT in polar solvents. This stability is related to physicochemical interactions between the permanent dipoles present on the surface of functionalized MWCNTs and the permanent dipoles from the solvent (dipole-dipole interactions between solvent-solute), as well as to the electrostatic repulsion between negatively charged carboxylic groups (coulombic solute-solute interactions).

MWCNTs with OCFs showed $\zeta$ potential values considerably higher, in modulus, than MWCNTs after OCF removal. This behavior can be explained by the presence of carboxylic groups in OCF structure. Since the OCFs are adhered to the MWCNT surface via physicochemical interactions, their functional groups increase MWCNT stability in polar solvents.

The MWCNTs functionalized at $70{ }^{\circ} \mathrm{C}, 20 \mathrm{~min}$ and $44 \mathrm{~mL}$ showed the more negative value of $\zeta$ potential $(-46.6 \pm 0.4 \mathrm{mV})$, which reinforces the optimal set of results for these conditions. The samples of MWCNTs functionalized with 88 and $20 \mathrm{~mL}$ of acid volume showed the most reduced values of $\zeta$ potential in modulus, $-44.0 \pm 0.7$ and $-43.7 \pm 0.4 \mathrm{mV}$, respectively, which can be related with a lower percentage of carboxylic groups, in accordance with the results of potentiometric titration.

MWCNTs with the presence of OCFs also resulted in lower HD values, which can be once more associated with the positive influence of carboxylic groups content in OCFs on its dispersion. MWCNTs submitted to treatments with acid volumes of 20 and $176 \mathrm{~mL}$, at $70^{\circ} \mathrm{C}$ and $20 \mathrm{~min}$, resulted in an increase of $\mathrm{HD}$ polydispersion and the occurrence of new intensity peaks of light scattering in distribution size analysis (the curves are shown in Figure S4, SI section). In the case of acid volume of $20 \mathrm{~mL}$, this phenomenon may be caused by a heterogeneous treatment related with the insufficient solid/liquid contact, with some MWCNTs that gained more functional groups than others. For the acid volume condition of $176 \mathrm{~mL}$, this behavior may be associated with MWCNTs of different aspect ratios, caused by a more aggressive ultrasonic cavitation in a short time interval (20 min).

\section{Conclusions}

An improved method for ultrasound-assisted functionalization of MWCNTs was established with remarkably reduction of acid volume, combined with moderate temperature of $70{ }^{\circ} \mathrm{C}$ and reaction time of only $20 \mathrm{~min}$. An expressive increase in the preservation of aspect ratio was achieved without compromising the functionalization extent, which has not been reported in the literature. A higher proportion of carboxylic groups and more negative zeta potential were also obtained. These results can be related with a more effective use of ultrasonic cavitation in reactions with reduced liquid volumes and a less harmful process by the control of temperature/time.

Therefore, a more efficient and environmentally friendly process was developed for MWCNT functionalization by a better comprehension of the solid/liquid interface and ultrasonic cavitation effects, leading to nanotubes with enhanced structural quality and more stable aqueous suspensions. This influence of ultrasonic cavitation in reduced volumes can be further studied in several chemical modifications of nanomaterials, which generally require a combination of appropriate preservation of their structure with high-efficiency process.

\section{Supplementary Information}

Supplementary information (percentage of each element by EA, extra SEM images, equations used for mean length determination of MWCNTs, DLS curves) is available free of charge at http://jbcs.sbq.org.br as PDF file. 


\section{Acknowledgments}

This research was supported by Petrobras. The authors also thank CNPq and FAPEMIG for the financial support, the Center of Microscopy of the UFMG for the electronic microscopy images and Professor Rubén Sinisterra for the use of electrophoretic mobility and DLS equipment.

\section{References}

1. de Volder, M. F. L.; Tawfick, S. H.; Baughman, R. H.; Hart, A. J.; Science 2013, 339, 535.

2. Mehra, N. K.; Jain, K.; Jain, N. K.; Drug Discovery Today 2015, 20, 750 .

3. Zhang, Q.; Huang, J. Q.; Qian, W. Z.; Zhang, Y. Y.; Wei, F.; Small 2013, 9, 1237.

4. Karousis, N.; Tagmatarchis, D.; Tasis, D.; Chem. Rev. 2010, $110,5366$.

5. Kim, S. W.; Kim, T.; Kim, Y. S.; Choi, H. S.; Lim, H. J.; Yang, S. J.; Park, C. R.; Carbon 2012, 50, 3.

6. Milowska, K. Z.; J. Phys. Chem. C 2015, 119, 26734.

7. Kabbani, M. A.; Tiwary, C. S.; Autreto, P. A. S.; Brunetto, G.; Som, A.; Krishnadas, K. R.; Ozden, S.; Hackenberg, K. P.; Gong, Y.; Galvao, D. S.; Vajtai, R.; Kabbani, A. T.; Pradeep, T.; Ajayan, P. M.; Nat. Commun. 2015, 6, DOI: 10.1038/ ncomms8291.

8. Chen, J.; Chen, Q.; Ma, Q.; J. Colloid Interface Sci. 2012, 370, 32.

9. Cho, J.; Boccaccini, A. R.; Shaffer, M. S. P.; Carbon 2012, 50, 3967.

10. Vennerberg, D. C.; Quirino, R. L.; Jang, Y.; Kessler, M. R.; ACS Appl. Mater. Interfaces 2014, 6, 1835.

11. Peng, K.; Liu, L. Q.; Li, H.; Meyer, H.; Zhang, Z.; Carbon 2011, 49, 70.

12. Morales-Lara, F.; Pérez-Mendoza, M. J.; Altmajer-Vaz, D.; García-Román, M.; Melguizo, M.; López-Garzón, F. J.; Domingo-García, M.; J. Phys. Chem. C 2013, 117, 11647.

13. Lobo, A. O.; Ramos, S. C.; Antunes, E. F.; Marciano, F. R.; Trava-Airoldi, V. J.; Corat, E. J.; Mater. Lett. 2012, 70, 89.

14. Wei, H.; Wei, Y.; Wu, Y.; Liu, L.; Fan, S.; Jiang, K.; Nano Res. 2013, 6, 208.

15. Martín, O.; Gutierrez, H. R.; Maroto-Valiente, A.; Terrones, M.; Blanco, T.; Baselga, J.; Mater. Chem. Phys. 2013, 140, 499.

16. Rasheed, A.; Howe, J. Y.; Dadmun, M. D.; Britt, P. F.; Carbon 2007, 45, 1072.

17. Liang, S.; Li, G.; Tian, R.; J. Mater. Sci. 2016, 51, 3513.

18. Zhou, W.; Sasaki, S.; Kawasaki, A.; Carbon 2014, 78, 121.

19. Samorì, C.; Sainz, R.; Ménard-Moyon, C.; Toma, F. M.; Venturelli, E.; Singh, P.; Ballestri, M.; Prato, M.; Bianco, A.; Carbon 2010, 48, 2447.
20. Avilés, F.; Cauich-Rodríguez, J. V.; Moo-Tah, L.; May-Pat, A.; Vargas-Coronado, R.; Carbon 2009, 47, 2970.

21. Ng, C. M.; Manickam, S.; Chem. Phys. Lett. 2013, 557, 97.

22. Maciejewska, B. M.; Jasiurkowska-Delaporte, M.; Vasylenko, A. I.; Kozioł, K. K.; Jurga, S.; RSC Adv. 2014, 4, 28826.

23. Wepasnick, K. A.; Smith, B. A.; Schrote, K. E.; Wilson, H. K.; Diegelmann, S. R.; Fairbrother, D. H.; Carbon 2011, 49, 24.

24. Castro, V. G.; Costa, I. B.; Lopes, M. C.; Lavall, R. L.; Figueiredo, K. C. S.; Silva, G. G.; J. Braz. Chem. Soc. 2017, 28, 1158.

25. Mazov, I.; Kuznetsov, V. L.; Simonova, I. A.; Stadnichenko, A. I.; Ishchenko, A. V.; Romanenko, A. I.; Tkachev, E. N.; Anikeeva, O. B.; Appl. Surf. Sci. 2012, 258, 6272.

26. Hanelt, S.; Orts-Gil, G.; Friedrich, J. F.; Carbon 2011, 49, 2978.

27. Chiang, Y. C.; Lin, W. H.; Chang, Y. C.; Appl. Surf. Sci. 2011, 257, 2401.

28. Wang, Z.; Shirley, M. D.; Meikle, S. T.; Whitby, R. L. D.; Mikhalovsky, S. V.; Carbon 2009, 47, 73.

29. Andrade, N. F.; Martinez, D. S. T.; Paula, A. J.; Silveira, J. V.; Alves, O. L.; Souza Filho, A. G.; J. Nanoparticle Res. 2013, 15, article ID 1761.

30. Li, Y.; Li, H.; Petz, A.; Kunsági-Máté, S.; Carbon 2015, 93, 515.

31. Pacheco, F. G.; Cotta, A. A. C.; Gorgulho, H. F.; Santos, A. P.; Macedo, W.A.A.; Furtado, C.A.; Appl. Surf. Sci. 2015, 357, 1015.

32. Wu, Z.; Mitra, S.; Carbon 2015, 88, 233.

33. Wu, Z.; Hamilton, R. F.; Wang, Z.; Holian, A.; Mitra, S.; Carbon 2014, 68, 686.

34. Rooze, J.; Rebrov, E. V.; Schouten, J. C.; Keurentjes, J. T. F.; Ultrason. Sonochem. 2013, $20,1$.

35. Ashokkumar, M.; Ultrason. Sonochem. 2011, 18, 864.

36. Vanhille, C.; Campos-Pozuelo, C.; Ultrason. Sonochem. 2012, $19,217$.

37. Sesis, A.; Hodnett, M.; Memoli, G.; Wain, A. J.; Jurewicz, I.; Dalton, A. B.; Carey, J. D.; Hinds, G.; J. Phys. Chem. B 2013, $117,15141$.

38. Likodimos, V.; Steriotis, T. A.; Papageorgiou, S. K.; Romanos, G. E.; Marques, R. R. N.; Rocha, R. P.; Faria, J. L.; Pereira, M. F. R.; Figueiredo, J. L.; Silva, A. M. T.; Falaras, P.; Carbon 2014, 69, 311.

39. Romanos, G. E.; Likodimos, V.; Marques, R. R. N.; Steriotis, T. A.; Papageorgiou, S. K.; Faria, J. L.; Figueiredo, J. L.; Silva, A. M. T.; Falaras, P.; J. Phys. Chem. C 2011, 115, 8534.

40. Bertoncini, M.; Hoepfner, J.; Pezzin, S.; Curr. Org. Chem. 2013, 17, 1838.

41. Mittal, G.; Dhand, V.; Rhee, K. Y.; Park, S. J.; Lee, W. R.; J. Ind. Eng. Chem. 2015, 21, 11.

42. Sahoo, N. G.; Rana, S.; Cho, J. W.; Li, L.; Chan, S. H.; Prog. Polym. Sci. 2010, 35, 837.

43. Ma, X.; Jia, L.; Zhang, L.; Zhu, L.; Chem. - Eur. J. 2014, 20 , 4072. 
44. Del Canto, E.; Flavin, K.; Movia, D.; Navio, C.; Bittencourt, C.; Giordani, S.; Chem. Mater. 2011, 23, 67.

45. Zhong, J.; Xie, T.; Deng, J.; Sun, X.; Pan, X.; Bao, X.; Wu, Z.; Chem. Commun. 2011, 47, 8373.

46. Hosur, M.; Barua, R.; Zainuddin, S.; Kumar, A.; Trovillion, J.; Jeelani, S.; J. Appl. Polym. Sci. 2013, 127, 4211.

47. Kim, Y. S.; Yang, S. J.; Lim, H. J.; Kim, T.; Park, C. R.; Carbon 2012, 50, 3315.

48. Rasband, W. S.; ImageJ, version 1.47; U. S. National Institutes of Health, Bethesda, Maryland, USA, 2013.

49. Lopes, M. C.; Castro, V. G.; Seara, L. M.; Diniz, V. P. A.; Lavall, R. L.; Silva, G. G.; J. Appl. Polym. Sci. 2014, 131, ID 41207.

50. da Silva, W. M.; Ribeiro, H.; Neves, J. C.; Sousa, A. R.; Silva, G. G.; J. Appl. Polym. Sci. 2015, 132, ID 42587.

51. Trigueiro, J. P. C.; Silva, G. G.; Lavall, R. L.; Furtado, C. A.; Oliveira, S.; Ferlauto, A. S.; Lacerda, R. G.; Ladeira, L. O.; Liu,
J.; Frost, R. L.; George, G. A.; J. Nanosci. Nanotechnol. 2007, 7, 3477.

52. Suryanto, B. H. R.; Fang, T.; Cheong, S.; Tilley, R. D.; Zhao, C.; J. Mater. Chem. A 2018, 6, 4686.

53. Clancy, A. J.; White, E. R.; Tay, H. H.; Yau, H. C.; Shaffer, M. S. P.; Carbon 2016, 108, 423.

54. Dong, B.; Su, Y.; Liu, Y.; Yuan, J.; Xu, J.; Zheng, L.; J. Colloid Interface Sci. 2011, 356, 190.

55. Sun, Z.; Nicolosi, V.; Rickard, D.; Bergin, S. D.; Aherne, D.; Coleman, J. N.; J. Phys. Chem. C 2008, 112, 10692.

56. Konkena, B.; Vasudevan, S.; J. Phys. Chem. Lett. 2012, 3, 867.

57. Coleman, J. N.; Adv. Funct. Mater. 2009, 19, 3680.

Submitted: February 11, 2019

Published online: July 22, 2019 